PROCEEDINGS OF THE

AMERICAN MATHEMATICAL SOCIETY

Volume 137, Number 1, January 2009, Pages 297-301

S 0002-9939(08)09482-3

Article electronically published on May 15, 2008

\title{
GENERALIZED KOSTANT CONVEXITY THEOREMS
}

\author{
PHILIP FOTH
}

(Communicated by Gail R. Letzter)

\begin{abstract}
We give a simple proof of the equality of the spectra for projections to Levi factors in the linear and non-linear cases, generalizing a classical theorem of Kostant.
\end{abstract}

\section{INTRODUCTION}

In this paper we give a simple proof of a result of Haines-Kapovich-Millson [4], which generalizes Kostant's linear and non-linear convexity theorems [6] to projections to Levi subgroups. In the complex case, we are going to use a theorem of Alekseev [1, which converts Poisson actions with moment maps in the sense of $\mathrm{Lu}$ [7] to the usual hamiltonian actions. Then, in order to establish the result in the real case, we employ certain involutions, the same way Evens and Lu used them in 2] to prove the Thompson conjecture for real reductive Lie groups.

Let us formulate the result; for our list of notation, please consult Section 2. To state the problems uniformly, we let $\mathbb{F}$ be either $\mathbb{R}$ or $\mathbb{C}, K$ be a suitable maximal compact subgroup of a reductive group $G(\mathbb{F}), N_{R}$ be the unipotent radical of a parabolic subgroup $R \subset G(\mathbb{F})$ and $M$ be its Levi subgroup; also let $K_{M}=K \cap M$.

Problem R1. Give conditions on $\lambda \in \mathfrak{a}^{+}$and $\mu \in \mathfrak{a}_{M}^{+}$that are necessary and sufficient for the orthogonal projection of $\operatorname{Ad}_{K}(\lambda)$ to contain $\operatorname{Ad}_{K_{M}}(\mu)$.

And now the non-linear counterpart:

Problem R2. Give conditions on $\lambda \in \mathfrak{a}^{+}$and $\mu \in \mathfrak{a}_{M}^{+}$that are necessary and sufficient in order that

$$
K \cdot e^{\lambda} \cdot K \cap N_{R} \cdot\left(K_{M} \cdot e^{\mu} \cdot K_{M}\right) \neq \emptyset .
$$

In this short paper we prove the following:

Theorem 1.1. The problems $R 1$ and $R 2$ are equivalent. That is, the conditions in Problem R1 are exactly the same as the conditions in Problem R2.

Received by the editors September 4, 2007, and, in revised form, January 7, 2008.

2000 Mathematics Subject Classification. Primary 53D20; Secondary 53D17, 22E15.

Key words and phrases. Reductive Lie group, parabolic subgroup, Poisson Lie group, moment map, convexity.

(C)2008 American Mathematical Society Reverts to public domain 28 years from publication 


\section{Notation}

We introduce the following notation:

$G$ - complex reductive Lie group with Lie algebra $\mathfrak{g}$

$B$ - a Borel subgroup with Lie algebra $\mathfrak{b}$

$N$ - the unipotent radical of $B$ with Lie algebra $\mathfrak{n}$

$\theta$ - a Cartan involution defining a maximal compact subgroup $K, \mathfrak{k}=\operatorname{Lie}(K)$

$H=B \cap \theta(B)$ - a Cartan subgroup with Lie algebra $\mathfrak{h}$

$G=K A N$ - Iwasawa decomposition, where $A \subset H$ has Lie algebra $\mathfrak{a}=\mathfrak{h}^{-\theta}$

$\Delta=\Delta(\mathfrak{g}, \mathfrak{h}), \Delta^{+}, \Sigma$ - systems of all roots, positive roots, simple roots

$T$ - maximal torus, $T \subset H$, with Lie algebra $\mathfrak{t}$

$G=K P$ - the Cartan decomposition, and on the Lie algebra level, $\mathfrak{g}=\mathfrak{k}+\mathfrak{p}$

$\langle\cdot, \cdot\rangle$ - an invariant non-degenerate pairing, extending the Killing form from $[\mathfrak{g}, \mathfrak{g}]$

Now, for a subset $I \subset \Sigma$ of positive roots:

$R \supset B$ - the standard parabolic subgroup corresponding to $I$

$N_{R}$ - the unipotent radical of $R$ with Lie algebra $\mathfrak{n}_{R}$

$M$ - a $\theta$-stable Levi subgroup, containing $H$, with Lie algebra $\mathfrak{m}$

$K_{M}$ - the maximal compact of $M$ contained in $K$, with Lie algebra $\mathfrak{k}_{M}$

$M=K_{M} A N_{M}$ - the Iwasawa decomposition, so $N=N_{R} \bowtie N_{M}$

$\mathfrak{n}_{M}$ - Lie algebra of $N_{M}$

$M=K_{M} P_{M}$ - the Cartan decomposition, on the Lie algebra level $\mathfrak{m}=\mathfrak{k}_{M}+\mathfrak{p}_{M}$

$\mathfrak{a}^{+}$- dominant chamber for $(\mathfrak{g}, \mathfrak{b})$

$\mathfrak{a}_{M}^{+} \supset \mathfrak{a}^{+}$- dominant chamber for $(\mathfrak{m}, \mathfrak{m} \cap \mathfrak{b})$.

\section{Complex CASE}

Let $\lambda \in \mathfrak{a}^{+}$be chosen. Let $\Phi(\lambda) \subset \mathfrak{a}_{M}^{+}$be the subset of those $\mu \in \mathfrak{a}_{M}^{+}$whose $K_{M}$-orbits occur in the projection $p: O_{\lambda} \longrightarrow \mathfrak{p}_{M}$. Here we denoted $O_{\lambda}=\operatorname{Ad}_{K}(\lambda)$ and have naturally identified $\mathfrak{k}^{*}$ with $\mathfrak{p}$ by using $\langle\cdot, \cdot\rangle$. If we put the coadjoint orbit structure on $O_{\lambda}$, then this projection becomes the moment map for the action of $K_{M}$. Note that $\Phi(\lambda)=p\left(O_{\lambda}\right) \cap \mathfrak{a}_{M}^{+}$. As a consequence of Kirwan's theorem [5], this intersection is a convex polytope $\Pi$ with vertices determined only by the simple roots in $I$.

In order to state the non-linear counterpart, we note that the following is straightforwardly true:

Lemma 3.1. The subgroup $K_{M} \subset K$ is a Poisson Lie subgroup with respect to the standard Poisson Lie group structure on $K$ defined by the Manin triple $(\mathfrak{g}, \mathfrak{k}, \mathfrak{a}+\mathfrak{n})$ with respect to $\operatorname{Im}\langle\cdot, \cdot\rangle$. There is a natural surjection $q: A N \longrightarrow A N_{M}$, which, by duality, is a Poisson map.

Let us now reformulate the problem R2 of [4] in the language of the dressing orbits. Take $\lambda \in \mathfrak{a}^{+}$and consider the dressing orbit $D_{\lambda}$ of $\exp (\lambda)$ by $K$ in $A N$. Using the action of $N_{R}$, each point of $D_{\lambda}$ can be moved to a unique point of $A N_{M}$. We refer to this operation as the non-linear projection $q: A N \longrightarrow A N_{M}$. In fact, as follows straight from the definitions, the projection $q: D_{\lambda} \longrightarrow A N_{M}$ is the Poisson moment map in the sense of Lu, for the Poisson action of $K_{M}$ on $D_{\lambda}$. By Alekseev's theorem [1, the linearization of this Poisson action is exactly the composition of the symmetrization map $a \mapsto a a^{*}$ followed by log. Therefore, if we consider the 
intersection of $q\left(D_{\lambda}\right)$ with $\exp \left(\mathfrak{a}_{M}^{+}\right)$, we will obtain exactly $\exp (\Pi)$, which proves Theorem 1.1 in the complex case.

\section{ReAl CASE}

We will treat the real case with the cunning use of involutions.

Let $\mathfrak{g}_{0}$ be a real form of $\mathfrak{g}$, defined by an involution $\tau$. Just as in [3], we can assume, after conjugating $\tau$, if necessary, that $\tau$ commutes with $\theta, \tau(\mathfrak{h})=\mathfrak{h}, \mathfrak{a}_{0}=\mathfrak{a}^{\tau}$ is maximal abelian in $\mathfrak{p}_{0}=\mathfrak{p}^{\tau}$, and that if a positive root $\alpha \in \Delta^{+}$restricts to a nonzero functional on $\mathfrak{a}^{\tau}$, then $\tau(\alpha) \in \Delta^{+}$. This leads to an Iwasawa decomposition $\mathfrak{g}_{0}=\mathfrak{k}_{0}+\mathfrak{a}_{0}+\mathfrak{n}_{0}$, where $\mathfrak{k}_{0}=\mathfrak{k}^{\tau}$ and $\mathfrak{n}_{0} \subset \mathfrak{n}$. Here and further we denote all the data from Section 2, pertaining to the chosen real form, by a subscript "0".

Now, let $R_{0}$ be a standard parabolic subgroup of $G_{0}$, with a Levi decomposition $R_{0}=M_{0} N_{R, 0}$, so that $M_{0}$ is $\theta$-stable and contains $\mathfrak{h}_{0}$. So, $M_{0}=M^{\tau}$, where $M$ is a Levi subgroup of the complexification $R$ of $R_{0}$, and $R_{0}=R^{\tau}$. Note that the unipotent radical, $N_{R}$, of $R$ is $\tau$-stable as well, and that $N_{R, 0}=N_{R}^{\tau}$.

In order to establish Theorem 1.1 in the real case, we first show that Problem R1 is equivalent for group $G_{0}$ and its complexification $G$. Recall the argument in 8 , Example 2.9], which states that for $\lambda \in \mathfrak{p}_{0}$, the fixed point set of $\tau$ on the $K$-orbit $O_{\lambda}$ coincides with the $K_{0}$-orbit $\operatorname{Ad}_{K_{0}}(\lambda)$, which we denote by $O_{\lambda}^{\tau}$. As before, denote by $p$ the projection $\mathfrak{p} \longrightarrow \mathfrak{p}_{M}$. Now, [8, Theorem 3.1] yields the following:

\section{Proposition 4.1.}

$$
p\left(O_{\lambda}^{\tau}\right) \cap \mathfrak{a}_{M}^{+}=\left(p\left(O_{\lambda}\right) \cap \mathfrak{a}_{0}\right) \cap \mathfrak{a}_{M}^{+} .
$$

Since $p\left(O_{\lambda}^{\tau}\right)=p\left(O_{\lambda}\right) \cap \mathfrak{p}_{0}$, any $\mu \in p\left(O_{\lambda}^{\tau}\right) \cap \mathfrak{a}_{M}^{+}$will also lie in $p\left(O_{\lambda}\right) \cap\left(\mathfrak{a}_{M}^{+}\right)^{\tau}$, and since the $\tau$-fixed point set of the $K_{M}$-orbit through $\mu$ will be exactly the $K_{M}^{\tau}$-orbit of $\mu$, we are done in the linear case.

Now we move to the non-linear case, where we need to show that problem R2 is equivalent for $G_{0}$ and $G$.

In the complex situation, there is a map $E: \mathfrak{p} \longrightarrow A N$, which goes as follows: for an element $X \in \mathfrak{p}$, we use the Cartan decomposition to consider $\exp (X)$ as a point of $G / K$, which is identified with $A N$ using the Iwasawa decomposition. Similarly, we define the map $E_{0}: \mathfrak{p}_{0} \longrightarrow A_{0} N_{0}$. The maps $E$ and $E_{0}$ are global diffeomorphisms.

Next, we recall the involution $\sigma: A N \longrightarrow A N$ from [2]. It is defined as follows:

$$
\sigma(b)=\operatorname{proj}_{A N}(\tau(b)),
$$

where $\operatorname{proj}_{A N}$ is the projection $G \longrightarrow A N$ given by the Iwasawa decomposition $G=A N K$.

If we let $\tau_{d}$ be given by $\operatorname{Ad}_{w_{0}} \tau$, where $w_{0}$ is the longest Weyl group element in the subgroup of $W$, generated by the reflections corresponding to the black simple roots in the corresponding Satake diagram for $\mathfrak{g}_{0}$, then the involution $\tau_{d}$ defines the quasi-split real form in the inner class of $\tau$. Another way of defining $\sigma$ is then:

$$
\sigma(b)=\dot{w}_{0} \cdot \tau_{d}(b), b \in A N,
$$

where $\cdot$ now means the dressing action of $\dot{w}_{0}$ on $A N$. Note that $\tau_{d}$ maps $A N$ to $A N$, so the action is well-defined.

We need to remark that $\sigma: A N \longrightarrow A N$ is a smooth involution, and $A_{0} N_{0}=$ $(A N)^{\sigma}$, but $\sigma$ only respects the group structure on $A N$ in the case when $\tau$ is already quasi-split. Now, [2, Lemma 4.4] says that 
Lemma 4.2. If $X \in \mathfrak{p}, Y \in \mathfrak{p}_{0}$, then

$$
E(\tau(X))=\sigma(E(X)) \text { and } E_{0}(Y)=E(Y) .
$$

Next, note that on the unipotent radical $N_{R}$, the involution $\sigma$ agrees with $\tau$, and therefore $N_{R}$ is $\sigma$-stable, and $N_{R, 0}=N_{R}^{\sigma}$. This means that the map $q$ is welldefined in the real case as well, and the definition is compatible with the complex case by means of $\sigma$. Now we claim that the real form $M_{0}$ of $M$ has analogous properties with respect to the restrictions of the involutions $\tau, \sigma$, and the maps $E$ and $E_{0}$. This is really a consequence of the fact that the simple roots that define the complex parabolic subgroup $R$ are taken from the Satake diagram for $\mathfrak{g}_{0}$, and this set $I$ is comprised of all the black simple roots and some white simple roots, where we include white roots in pairs if they are connected by an arrow. Let us summarize these properties in the list below.

- We have $M=K_{M} A N_{M}$ and $M_{0}=K_{M, 0} A_{0} N_{M, 0}$, where $K_{M, 0}=K_{M}^{\tau}$, $A_{0}=A^{\tau}$, and $N_{M, 0}=N_{M}^{\sigma}$.

- For $g \in \mathfrak{p}_{M}, E_{\mid \mathfrak{p}_{M}}(g)=E(g)$ and thus Lemma 4.2 applies.

- If $g \in A N, g=n m$ for $n \in N_{R}$ and $m \in A N_{M}$, then $\sigma(g)=\sigma(n) \sigma(m)$.

The last property is a simple consequence of the definition of $\sigma$. Now we can show that

Lemma 4.3. For $\lambda \in \mathfrak{a}_{0}^{+}$,

$$
q\left(D_{\lambda}^{\sigma}\right)=\left(q\left(D_{\lambda}\right)\right)^{\sigma} .
$$

Proof. The inclusion of the right hand side into the left hand side is obvious. Now let $g=q(b)$ for $b \in D_{\lambda}^{\sigma}$. Decompose $b=n_{1} n_{2}$ for $n_{1} \in N_{R}, n_{2} \in A N_{M}$. According to the above, since $\sigma(b)=b$, then from the uniqueness of $N_{R}$, it follows that $n_{1} \in N_{R, 0}$ and $n_{2} \in A_{0} N_{M}^{\sigma}$. Thus, $q(b)=n_{2}$ and we are done.

For visualization, we present the following commutative cube:

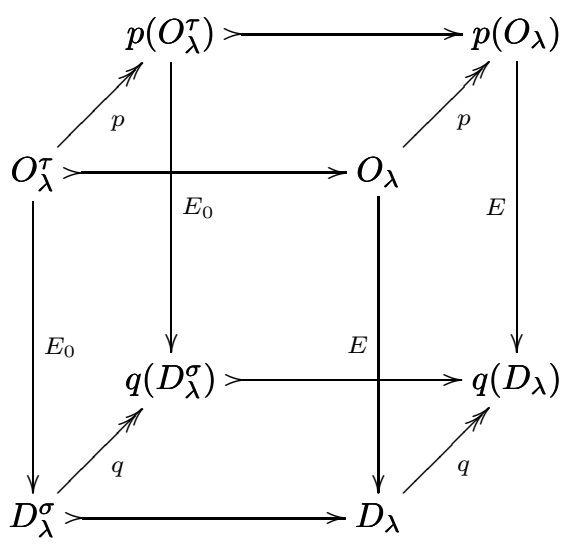

The commutativity of the right face of the cube is by Alekseev [1], the front and back faces are by Evens-Lu [2], the top face is by O'Shea-Sjamaar [8], and the main property for the bottom face is explained in the previous lemma.

This finishes the proof of Theorem 1.1. 


\section{ACKNOWLEDGements}

The idea of this proof came during John Millson's lecture on [4, and I thank him for that. I also thank Misha Kapovich for showing me a preliminary version of the introduction to 4 and the referee for useful comments.

\section{REFERENCES}

[1] A. Alekseev. On Poisson actions of compact Lie groups on symplectic manifolds. J. Diff. Geom., 45: 241-256, 1997. MR1449971 (99b:58086)

[2] S. Evens and J.-H. Lu. Thompson's conjecture for real semisimple Lie groups. The breadth of symplectic and Poisson geometry. Progr. Math., 232: 121-137, Birkhäuser Boston, 2005. MR2103005 (2006c:22013)

[3] P. Foth and J.-H. Lu. A Poisson structure on compact symmetric spaces. Comm. Math. Phys., 251: 557-566, 2004. MR2102330 (2005g:53156)

[4] T. Haines, M. Kapovich and J. Millson. Ideal quadrilaterals in Euclidean buildings, constant term maps for spherical Hecke rings and branching to Levi subgroups. Preprint, 2005.

[5] F. Kirwan. Convexity properties of the moment mapping. III. Invent. Math., 77: 547-552, 1984. MR759257 (86b:58042b)

[6] B. Kostant. On convexity, the Weyl group and the Iwasawa decomposition. Ann. Sci. École Norm. Sup., 6: 413-455, 1973. MR0364552 (51:806)

[7] J.-H. Lu. Momentum mappings and reduction of Poisson actions. Symplectic geometry, groupoids, and integrable systems: 209-226, MSRI Publications 20, Springer, New York, 1991. MR.1104930 (92j:58036)

[8] L. O'Shea and R. Sjamaar. Moment maps and Riemannian symmetric pairs. Math. Ann., 317: 415-457, 2000. MR1776111 (2001g:53146)

Department of Mathematics, University of Arizona, Tucson, Arizona 85721-0089

E-mail address: foth@math.arizona.edu 